\title{
Structural and mechanical properties of new sizing compositions based on natural and synthetic water-soluble polymers
}

\author{
Salokhiddin Mardonov1,* and Khasan Saidov \\ ${ }^{1} \mathrm{PhD}$, Department of Textile technology and design, Bukhara Engineering Technological \\ Institute, Bukhara, Uzbekistan \\ ${ }^{2}$ student, Department of Light Industry Machinery and Equipment, Bukhara Engineering \\ Technological Institute, Bukhara, Uzbekistan \\ *E-mail: saloxiddin.1980@mail.ru
}

\begin{abstract}
In the article results of studying specific structurally-mechanical properties new developed sizing compositions on the basis of corn starch and water-soluble synthetic polymeric preparations such as K-4 and uniflock made in Company "Navoiazot" are given. It is established that, introduction in starch pastes acrylates leads to increase coefficient of thixotropic restoration, i.e. to increase of speed relaxation processes.
\end{abstract}

Keywords: size, starch, adhesion, thixotropic, rheology, yarn, fluidity limit, paste, retrogradation, structuring

\section{Introduction}

Structural-mechanical or rheological properties for fluid systems means the behavior of polymer systems upon deformation. They determine the dependences linking stresses at different temperatures and modes of deformation and deformation of polymer systems, and provide valuable information about their properties, structure, and structural transformations. They are of great importance not only from the point of view of the study of the systems themselves and the study of changes occurring in them, but also from the point of view of the problems associated with the use of such systems in technological processes [1,2].

In the processes of sizing textile materials, the used sizing compositions undergo various mechanical influences, which change the rheological properties of sizing systems based on starch and water-soluble acrylates [3]. It can be expected that due to the complexation reaction of starch and acrylic polymers, the rheological properties of the systems undergo significant changes.

The advantage of polyacrylate compositions as a sizing preparation lies in its high adhesive property. In addition, polyacrylates can be obtained of homopolymer and copolymer character, with various derivatives of acrylic and methacrylic acids, which allows combining different ratios of monomers in a polymer molecule and obtaining a wide range of physical and chemical properties in the synthesized material [4]. 


\section{Materials and methods}

On the basis of these studies, we have developed a new dressing composition based on corn starch obtained from secondary raw materials, as well as synthetic water-soluble polymer preparations such as K-4 and uniflock produced at Navoiazot OJSC. The introduction of acrylic polymers into the composition of the shrinking agents leads to a change in the structural and mechanical properties.

Polymer solutions, including starches, are not structureless. The structure of solutions is understood as the mutual arrangement of the solvent and polymer molecules, the conformation of macromolecules, and the interaction between polymer macromolecules [5]. The stability of the structure can be judged by the values of the degree of thixotropic reduction given in Table 1 . Thixotropy - the ability of structures after their destruction as a result of some mechanical action is spontaneously restored in time, that is, thixotropy is the ability to reversible isothermal transformation of a sol into a gel. Table 1 shows that starch pastes containing K-4 and unifloc are characterized by higher values of the degree of thixotropic reduction.

Table 1. Yield point and degree of thixotropic reduction of starch solutions with different contents of K-4 and unifloc.

\begin{tabular}{lllll}
\hline $\begin{array}{l}\text { Composition and content of components } \\
\text { in solution }\end{array}$ & $\begin{array}{l}\text { Yield point } \\
(\mathrm{Pa})\end{array}$ & $\begin{array}{l}\text { Degree of } \\
\text { thixotropic } \\
\text { recovery, } \%\end{array}$ \\
\cline { 1 - 1 } $\begin{array}{l}\text { Starch, } \\
\%\end{array}$ & $\begin{array}{l}\text { K-4, } \\
\%\end{array}$ & $\begin{array}{l}\text { Uniflock, } \\
\%\end{array}$ & & \\
\hline 6 & - & - & 3,89 & 88,57 \\
6 & 0.2 & 0.2 & 11.12 & 90.12 \\
6 & 0.3 & 0.2 & 12.23 & 91.79 \\
6 & 0.4 & 0.2 & 20.57 & 93.52 \\
6 & 0.5 & 0.2 & 30.58 & 97.16 \\
6 & 0.6 & 0.2 & 42.6 & 98.4 \\
6 & 0.5 & 0.3 & 36.44 & 97.3 \\
6 & 0.5 & 0.4 & 42.15 & 98.4 \\
6 & 0.5 & 0.5 & 46.83 & 98.8 \\
\hline
\end{tabular}

Such a gradual restoration of the structure and, consequently, an increase in its strength occurs not only when the system is at rest, but also when the system flows at a rate lower than that which caused the given degree of destruction of the original structure. However, with the reverse transition from a steady flow with a high velocity to a flow with a lower velocity, some recovery of the structure occurs and, accordingly, the effective viscosity and strength of the structure increase, and the higher the content of K-4 in the system, this effect is more pronounced. 
Thus, the introduction of K-4 starch and uniflock into the paste leads to an increase in the coefficient of thixotropic recovery, i.e., an increase in the rate of relaxation processes. Due to their high adhesive properties, polyacrylate compositions are becoming increasingly important as a sizing agent for sizing cotton fibers [6].

\section{Results}

A change in the chemical composition of these compounds allows a wide variation of their properties, which is much more difficult to accomplish with polymers of other groups. Therefore, this work presents the results of experimental substantiation of the influence of K-4 and uniflock, which is part of the sizing compositions, on the structural and mechanical properties of starch solutions.

The study of the rheological properties of $6 \%$ glues based on corn starch with the addition of small amounts $(0.2-0.6 \%)$ of K-4 and uniflock showed that the rheological properties of the systems change significantly when these preparations are introduced.

Table 2 shows the change in the viscosity of $6 \%$ starch pastes (at $\gamma=27 \mathrm{~cm}-1$ ) from the content of the introduced K-4 at different temperatures.

Table 2. Viscosity changes of $6 \%$ starch paste from content of K-4 in it at different temperatures.

\begin{tabular}{llllll}
\hline Temperature, $\mathrm{K}$ & \multicolumn{5}{l}{ The viscosity of the dressing (Pa.s) at various concentrations of K-4, } \\
& $\%$ & 0.3 & 0.4 & 0.5 & 0.6 \\
& 0.2 & 0.67 & 1.15 & 1.48 & 1.93 \\
298 & 0.35 & 0.58 & 1.03 & 1.22 & 1.60 \\
313 & 0.26 & 0.50 & 0.91 & 1.13 & 1.45 \\
323 & 0.20 & 0.41 & 0.83 & 1.05 & 1.32 \\
333 & 0.15 & 0.35 & 0.77 & 0.93 & 1.24 \\
343 & 0.12 & 0.30 & 0.74 & 0.84 & 1.18 \\
353 & 0.07 & & & & \\
\hline
\end{tabular}

From Table 2 it follows that the addition of K-4 to the starch system causes an increase in the viscosity of the system. This fact indicates the reaction of complexation of K-4 with starch, since in the polymer chain of starch (more precisely, its constituents - amylose and amylopectin), hydroxyl groups are likely to be present in a position convenient for complexation (at a distance of $2.42 \AA$ at an angle of 1090).

The formation of complexes is possible both within one polymer chain and between two chains [7]. The formation of such knots can lead to a decrease in the mobility of the chains, i.e. limiting their thermal movement, increasing the structuredness of the system and the formation of a more rigid chain, and as a result of all this, to an increase in the viscosity of the system.

From table 2 it also follows that for all systems with increasing temperature, the viscosity decreases. This can be explained by the fact that as the temperature rises, the energy of the thermal 
motion of the polymer units sharply increases and, at certain values, this energy increases the energy of intra-intermolecular interaction.

Starch solutions that have stood for a long time are capable of aging due to retrogradation and biodegradation phenomena. The ability to retrograde is extremely strongly manifested in solutions of pure amylose, which aggregates over time and finally forms an insoluble microcrystalline precipitate [8].

The figure 1 shows the data of the study of the aging process of $6 \%$ starch paste with $\mathrm{K}-4$ $(0.5 \%)$ uniflock $(0.3 \%)$ and without it at a temperature of $293 \mathrm{~K}$ by changing the viscosity of the system (at $\gamma=27 \mathrm{~s}-1)$.

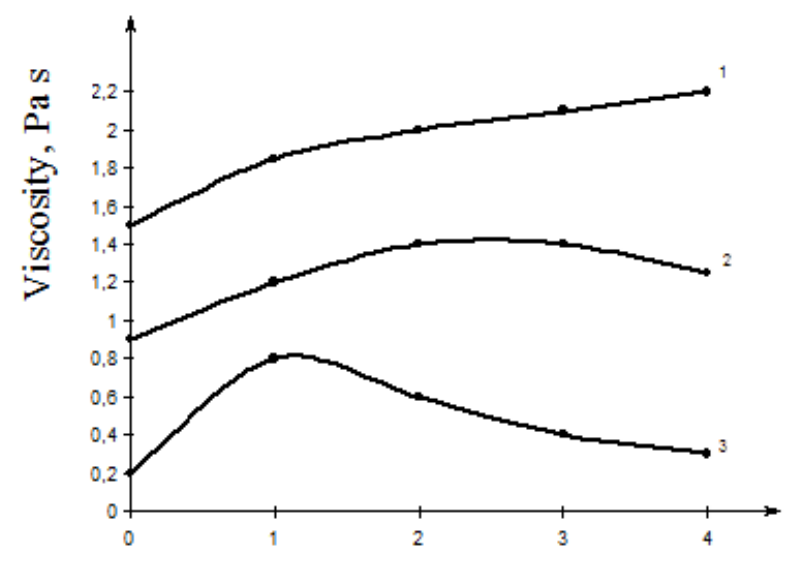

Aging time, days $\tau$.
Figure 1. Kinetics of the "Aging" process of $6 \%$ starch paste with K-4 (1), uniflock (2), without it (3).

It follows from the figure 1 that for starch paste that does not contain K-4 (curve 1), the dependence of the paste viscosity on the storage time passes through a maximum. The presence of a maximum in this dependence can be explained by the occurrence of two processes: an increase in the viscosity of the paste during the first day is a consequence of the process of retrogradation of the paste, i.e., the aggregation of amylose macromolecules. In contrast to curve 3, curves 1 and 2 , which characterize the dependence of the viscosity of $6 \%$ starch paste, with K-4 and uniflock does not have a maximum.

The viscosity of this paste increases monotonically within 5 days. The absence of a decrease in the viscosity of the paste on the second day of storage is explained by the blocking of the process of microbiological destruction of starch due to the antiseptic action of K-4 and uniflock. This can be judged by the angle of inclination to the abscissa axis of the curves of viscosity versus storage duration. At the same time, we studied the periods during the first day when the process of microbiological destruction does not leave its mark.

It follows from the figure 1 that the slope of curve 1 is much greater than the slope of curve 2, which indicates a slowdown in the process of retrograding of starch pastes in the presence of K-4 
and uniflock, i.e., polyacrylates have a stabilizing effect on starch pastes, slowing down the processes retrogradation of starch pastes and microbiological destruction of starch.

\section{Conclusion}

Thus, significant changes in the rheological properties of solutions of the polymer composition based on corn starch, K-4 and uniflock indicate a good sizing ability of cotton yarn.

\section{References}

[1] Ganzyuk, L.I. New preparations in sizing technology / L.I. Ganzyuk // Technics. - 1991. - P. 223.

[2] Mardonov, Salokhiddin. 2020 Analysis of quality indicators of sizing warp threads / Salokhiddin Mardonov, Sanovar Khamraeva, Kodir Muminov, Khakim Rakhimov and Elyor Kuldoshev // International Journal of Advanced Science and Technology. - № 4. - P. 4957-4968.

[3] Mardonov, S.E. Structural and mechanical properties of a water-soluble synthetic acrylic polymer and new abrasive compositions based on uzhitan / S.E. Mardonov, M.S. Norova, M.B. Ayupova // Scientific and technical journal of science and technology development. 2020. - № 2. - P. 32-37.

[4] Abrorov, A. Physical essence and process of laser hardening of circular saw of a saw cylinder / A. Abrorov, M. Gapparova, A. Temirov and N. Mirzakulova // International Journal of Advanced Research in Science, Engineering and Technology. - 2020. - № 7(1). - P. 1241812421.

[5] Latipova, I.M. Effect of mechanical treatment on the technological properties of starch gluing / I.M. Latipova, I.L. Sedova, N.A. Ermolaeva, V.A. Padokhin, A.P. Moryganov // Textile chemistry. - 1993. - № 1(3). - P. 26-30.

[6] Amonov, M.R. Water-soluble polymer compositions based on local raw materials for use in the production of cotton fabrics and the technology for their production: Diss ... Doctor of Engineering Sciences / M.R. Amonov. - Tashkent, 2005, 252 p.

[7] Ikhtiyarova, G.A. On the processes of saponification of acrylic emulsion in order to obtain a thickener for printing fabrics Uzbek / G.A. Ikhtiyarova, O.M. Yariev, B.A. Mavlonov, A.A. Khaidarov // Chemical journal. - 2002. - № 3. - P. 77-81.

[8] Alekseeva, S.V. Modification of the properties of hydrogels and starch films with specialized enzyme preparations / S.V. Alekseeva, S.A. Koksharov // Chemistry and chemical technology. - 2003. - № 46(1). - P. 120. 